\title{
oints
}

$\sqrt{2}$

\section{Trapeziometacarpal osteoarthritis: pyrocarbon interposition implants}

\author{
SIMONA ODELLA ${ }^{1}$, AMOS M. QUERENGHI ${ }^{1}$, ROBERTA SARTORE ${ }^{2}$, AGOSTINO DE FELICE ${ }^{1}$, \\ UGO DACATRA ${ }^{1}$
}

${ }^{1}$ Division of Hand Surgery, Gaetano Pini Orthopaedic Institute, Milan, Italy

2 Clinical Institute Sant’Ambrogio, Milan, Italy

\section{Abstract}

Purpose: the aim of this study was to evaluate the effectiveness of interposition arthroplasty of the trapeziometacarpal (TMC) joint with pyrolitic carbon implants for the treatment of TMC osteoarthritis.

Methods: we evaluated two groups of patients surgically treated for TMC osteoarthritis: group 1 (34 patients - 36 TMC joints) treated with PyroDisk implantation and group 2 (25 patients - 25 TMC joints) treated with the Pyrocardan implant. All these patients were clinically evaluated at follow-up using the DASH score, Mayo Wrist score and VAS pain score.

Results: the mean follow-up was 42 months in group 1 and 12 months in group 2. Both groups showed good clinical outcomes in terms of pain relief, range of motion, and pinch and grasp strength. Revision surgery was needed in only one case in group $1(2.8 \%)$ and in three cases (12\%) in group 2.

Conclusions: prosthetic replacement of the TMC joint was found to be a good solution for low-demand patients. However, the PyroDisk could be a good solution in selected patients (Eaton stage I-III, non-subluxated joint): it provides good pain relief, good range of motion, good pinch and grasp strength, and stable results at more than three-years of follow-up.

Level of evidence: Level IV, therapeutic case series.

Key Words: implant, interposition arthroplasty, osteoarthritis, pyrocarbon, trapeziometacarpal.

\section{Corresponding Author:}

Simona Odella, MD

Division of Hand Surgery, Gaetano Pini

Orthopaedic Institute

Piazza Cardinale Andrea Ferrari 1, 20122 Milan, Italy

E-mail: simonettaodella@gmail.com

\section{Introduction}

Trapeziometacarpal (TMC) arthritis is a frequent pathology; women aged 50 years and over are the most affected population. Pain at the base of the thumb associated with loss of strength are the most common symptoms, leading to limitation in daily activities.

Several factors lead to TMC arthritis (articular surface congruity, ligamentous instability), although the exact cause of the pathology is still unclear.

In the literature there is no consensus on whether arthritis affects mainly the most or the least congruous joints. Indeed, some authors support the theory that the least congruous joints deform and wear with adaptation to the localized stress (1-3); others argue the most congruous joints are the ones more affected by TMC arthritis (4).

Ligaments have a key role in joint stability and degeneration, but, once again, there is no consensus in the literature on which might be considered the most important in joint stability; some authors consider the anterior oblique ligament to be the most important (57), while others have stated that the dorsal ligament complex (dorsolateral ligament and posterior oblique ligament) has this role (8).

Eaton, in 1987, compiled a classification comprising four different progressive radiological stages of TMC osteoarthritis. Even though this classification continues to be used, its value is limited given that the clinical condition is often not correlated to radiographic images.

Furthermore, radiological examination does not allow evaluation of the cartilaginous surface, which is one of the most important aspects to take into account when deciding the kind of treatment. Only arthroscopy can evaluate the condition of the cartilage and ligaments in order to correctly stage the pathology, but it is an invasive procedure and sometimes difficult to perform (9). 
In the early stages of TMC osteoarthritis, the treatment is non-surgical, based on the use of a cast during the night, anti-inflammatory drugs (NSAIDs), local physical therapy, and intra-articular injections of a steroidal product or hyaluronic acid; unfortunately, these treatments are palliative and often, after an initial period of remittance of pain, the patient reports a worsening of the symptoms. If all these conservative treatments fail, surgery remains the only possible treatment.

Many surgical treatments are available - trapeziectomy, trapeziectomy and ligament reconstruction, arthrodesis, arthroscopic resection, metacarpal osteotomy, prosthetic implant arthroplasty - but there is currently no gold standard. Theoretically, the last of the above options allows preservation of joint anatomy and biomechanics (10).

The development of surgical treatment with arthroplasty dates back to the 1960s. The first implant used was the Swanson silicon endoprosthesis (11). Since this treatment showed poor outcomes and a high rate of complications, as reported in several studies (12$15)$, this procedure was limited to low-demand patients affected by rheumatoid arthritis (16).

This kind of prosthesis was followed by the development of several metal total joint implants (ball-andsocket prostheses) which showed good results (17). However, the main problem with these implants was radiographic loosening of the trapezial component $(18,19)$.

In more recent years, to avoid this complication, a new material was introduced for TMC implants: pyrolitic carbon. The most important advantage of this inert material is that it has a module of elasticity similar to that of cortical bone. Different pyrolitic carbon devices have been developed and used to treat TMC osteoarthritis, above all Eaton stages I or II $(20,21)$, with good clinical outcomes.

The aim of this study was to clinically evaluate patients submitted to a surgical treatment for TMC, namely interposition arthroplasty with pyrolitic implants, in order to assess the effectiveness of this kind of treatment in this pathology.

\section{Methods}

In this study, we evaluated patients surgically treated with TMC interposition arthroplasty with pyrolitic implants from 2007 to 2014 in our Hand Surgery Unit.

Patients suffering from symptomatic TMC osteoarthritis (pain, disability and loss of strength) for more than three years were included in the study. The diagnosis of TMC osteoarthritis was confirmed in all cases by an X-ray of the hand and their TMC ostheoarthritis was graded using the Eaton scale. All the patients underwent surgical treatment only after failure of the conservative approach.

Fifty-nine patients were enrolled in the present study and divided into two groups.

Group 1 included 34 patients (34 TMC joints treated) who underwent trapezium replacement with PyroDisk (Integra Life Sciences, Plainsboro, NJ). PyroDysk is a non-anatomical interposition implant, made of pyrocarbon. It is a biconcave disk with a central hole to allow stabilization with a tendon (Fig. 1). In order to implant PyroDisk a longitudinal skin incision at the TMC joint is made. Then the radial artery and the sensory branches of the radial nerve are identified and protected. After performing the capsulotomy, a bone resection of the base of the first metacarpal and of the distal part of the trapezium are performed.Then, with a specific tool, the space between trapezium and the metacarpal bone is prepared for insertion of the implant. To stabilize the implant in the joint, a strip of the ulnar part of flexor radialis carpi is passed through the hole made in the base of the metacarpal bone, the hole in the PyroDisk and the hole made in the trapezium.

Group 2 included 25 patients (25 TMC joints treated) who underwent trapezium replacement with Pyrocardan (Tornier, Montbonnot Saint Martin, France). Pyrocardan is a pyrolitic anatomical interposition implant (biconcave-shaped) (Fig. 2). The dorsal approach to the TMC joint is used, since it allows easier access to and resection of the trapezium ostheophytes. A minimal bone resection at the base of the first metacarpal and at the distal part of the trapezium is performed. In performing the bone resection, it must be remembered that the longitudinal axis of the metacarpal and that of the trapezium have to lie on the same line (co-axiality of the two bones) and the line of resection must be perpendicular to this and, at the same time, must spare the anatomical convexity of the articular surface of both bones. In this way, the Pyrocardan can be implanted without the need for a stabilization system. 

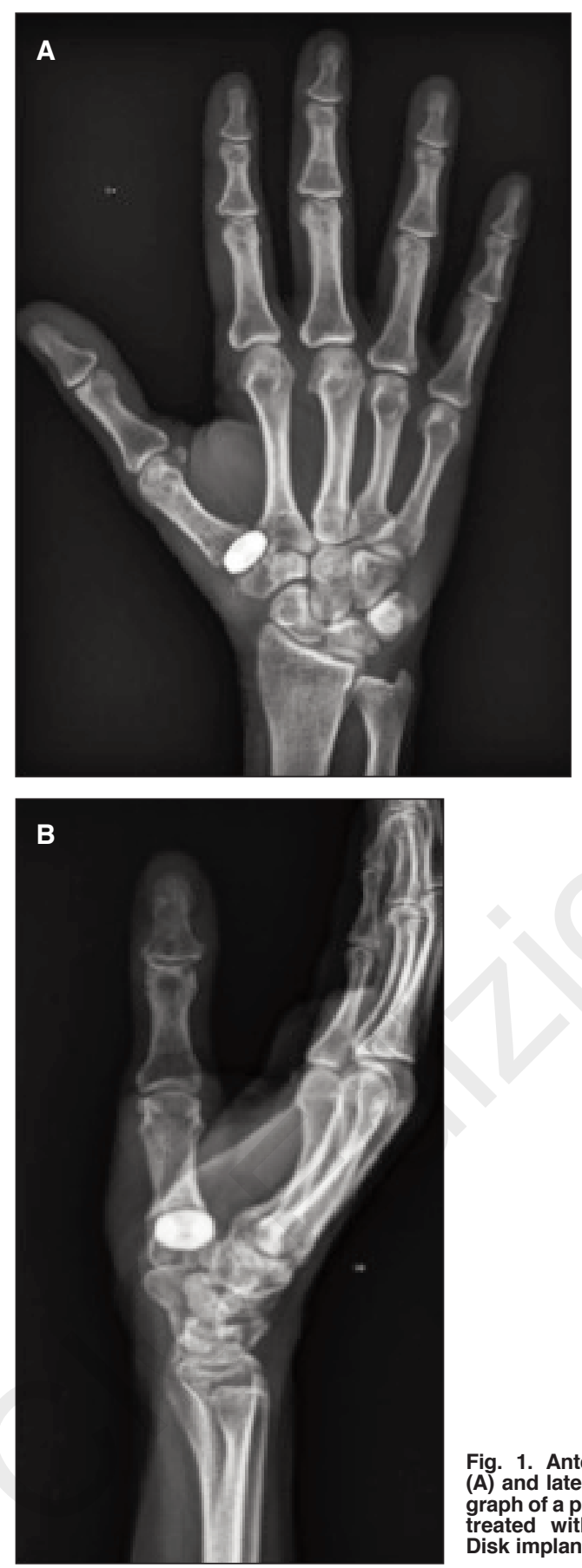

Fig. 1. Anterioposterior (A) and lateral (B) radiograph of a patient's hand treated with the PyroDisk implant.

All the patients in both groups were clinically evaluated using the DASH score and the MayoWrist score; pain was evaluated using a VAS scale. Furthermore, an X-ray of the hand was taken at the follow-up evaluation.

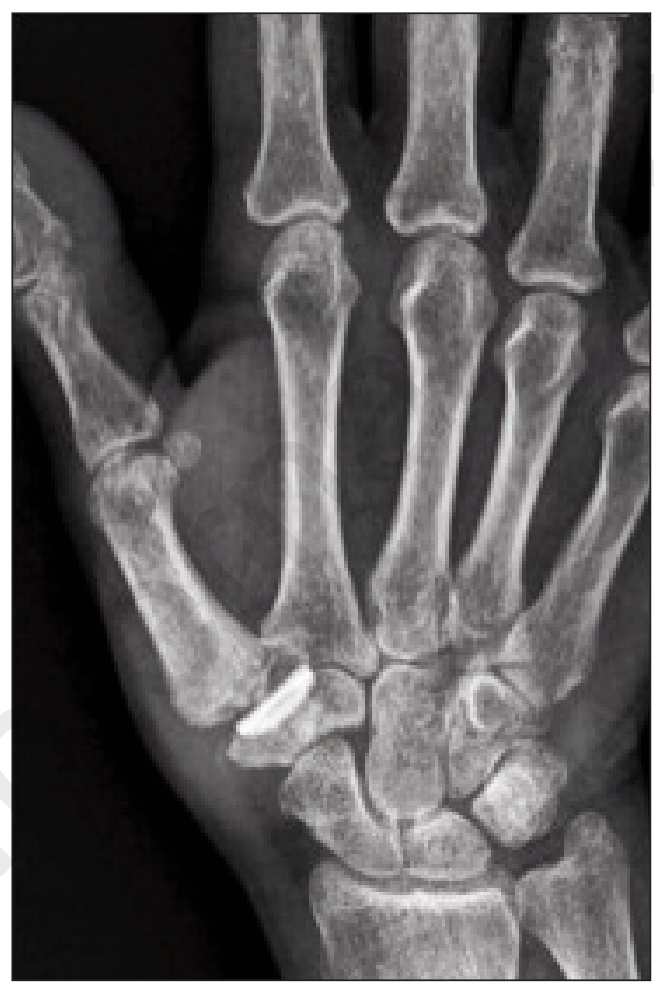

Fig. 2. An example of a patient treated with the Pyrocardan implant.

\section{Results}

Group 1 included patients with Eaton stage I-III disease, without first metacarpal subluxation. The mean age of the group 1 patients was 62 years (range 51-70 years). They had a mean follow-up of 42 months (range 12-84 months). They recorded a mean VAS pain score of 3.3, a mean DASH score of 19.2, and a mean Mayo Wrist score of 80.6. They showed a 20\% of reduction of strength in the surgically treated joint compared with the contralateral one. In one case we had to remove the implant due to persistent pain. In another patient implant subluxation was observed although the patient experienced no referred pain or limitation in daily activities.

Group 2 included patients with Eaton stage I-III disease, with first metacarpal subluxation. The patients in this group had a mean age of 55 years (range 50-65 years). They were followed up for an average of 12 months (range 12-24 months). Their average VAS pain score was 4 and they recorded a mean DASH score of 22.4 and a mean Mayo Wrist score of 70.5. 
They, too, showed a $20 \%$ reduction of strength in the surgically treated joint compared with the controlateral one. In this group, $12 \%$ of the patients were not satisfied, reporting pain that was worse than before the surgical treatment; in two cases we had to remove implant due to persistent pain and stiffness (Fig. 3). Furthermore, it has recently emerged that we need to remove another one.

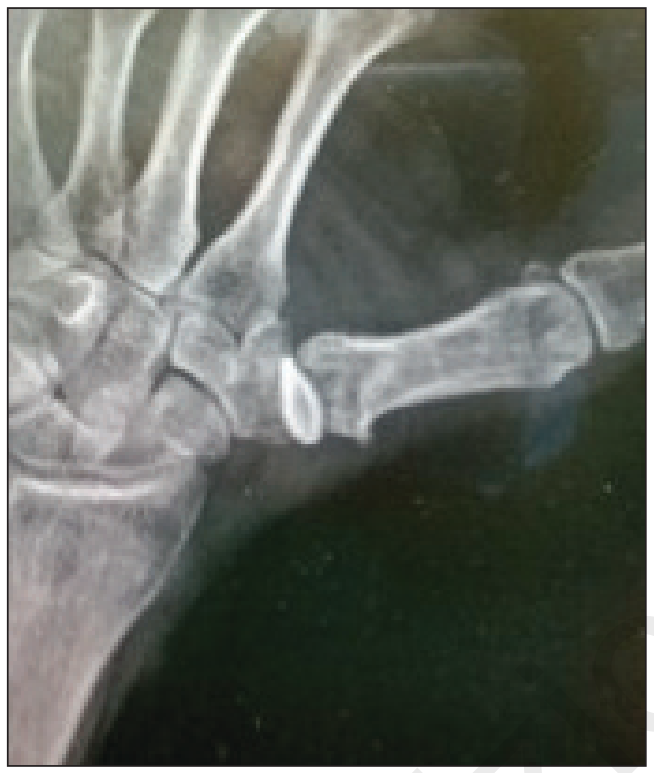

Fig. 3. The radiograph shows incorrect bone resection; the medial ostheophyte has not been resected and the implant is too small.

\section{Discussion}

Surgery for TMC osteoarthritis is the only treatment able to provide pain relief and restore a good range of motion and good pinch and grasp strength with stable results over the years.

However, since there is currently no consensus on the most suitable surgical treatment, there are different surgical techniques that can be used: trapeziectomy, trapeziectomy and ligament reconstruction, arthrodesis, arthroscopic resection, metacarpal osteotomy, and prosthetic implant arthroplasty. Of all these techniques, the last one (prosthetic arthroplasty) is the one that can offer preservation of joint anatomy and biomechanics. Over the last 50 years, several prostheses have been developed. The first model was the silicon one by Swanson (11), which, however, due to a high rate of complications, was indicated only in patients suffering from reumathoid arthritis. Therefore, different materials were used and numerous metal total joint implants were developed and used for the treatment of TMC arthritis. The earliest, designed by De la Caffenière and Aucouturier, was a cemented ball-andsocket implant with a polyethylene cup inserted in the trapezium and a cobalt-chromium stem in the first metacarpal. The main problem with this implant was radiographic loosening of the trapezial component $(18,19)$. De Smet et al. (22) reported on 43 arthroplasties performed using the De la Caffenière prosthesis: the patient satisfaction rate was $70 \%$ and the patients showed a good range of motion, although loosening occurred in $44 \%$ of the implants; these authors found a relationship between younger age and loosening. In recent years new metal prostheses have been implanted. Regnard et al. (17) reported on 100 patients treated with a metal bone-and-socket semimodular unconstrained cementless hydroxyapatitecoated prosthesis (Electra). They reported good results in terms of improvements in strength, range of motion and pain relief, but observed loosening of the cup, subsidence and dislocation of the distal component in $15 \%$ of cases. Cooney et al. (23) developed a cemented implant with a metal pedestal with a sphere in the trapezium, and a polyethylene stem in the first metacarpal. The patients showed excellent motion and pinch strength, but $36 \%$ of the implants developed heterotopic ossifications after surgery and this condition had an adverse impact on functional outcome.

In recent years a new material has been used for TMC implants, namely pyrolytic carbon, a synthetic material formed by pyrolysis of a hydrocarbon gas. Since it has a module of elasticity similar to that of cortical bone, this material appeared suitable for interposition arthroplasty. Different implants made from this material are available for interposition arthroplasty.

In our study we used an anatomically shaped one (Pyrocardan) and a non-anatomically shaped one (PyroDisk). As reported in the literature (21), we observed, during the follow-up, good outcomes in patients treated with PyroDisk, with a low rate of complications, and particularly of revisions. Indeed, we had only one case in which the PyroDisk had to be removed because of persistent pain and functional impairment.

Even though Iogoe et al. (24) recently reported better results in patients treated by trapeziectomy than in ones treated by interposition implant, we believe that 
the PyroDisk is a good solution in selected patients (Eaton stage I-III disease without subluxation); it provides good pain relief, a good range of motion, good pinch and grasp strength, and stable results at more than three years' follow-up. Conversely, we had good outcomes, but a higher rate of complications, when using Pyrocardan. Even though Bellemere et al. (20) reported excellent improvements in pain and subjective scores with no complications and no need for revision surgery with this type of implant, we had to remove the Pyrocardan implant in three cases $(12 \%)$ because of persistent pain and functional impairment. We think that this difference in complication rates could be due to differences in surgical technique. In fact, compared with PyroDisk implantation, Pyrocardan implantation is more difficult because, in order to ensure correct load transmission, it demands greater precision in bone resections (the longitudinal axis of the first metacarpal must be the same as that of the trapezium, and the resection must spare the anatomical convexity of the articular surface).

In conclusion, TM arthritis treatment is still a controversial topic. Considering the good outcomes obtained (pain relief, good range of motion, good pinch and grasp strength), which were stable over the first three years of follow-up, and the low rate of complications, we argue that PyroDisk could be considered, in the short term, as a valid possibility for treating TMC osteoarthritis in patients presenting with Eaton stage I-III disease and a non-subluxated joint. If opting for a Pyrocardan implant the surgeon must remember that this prosthesis needs more precise bone resections to avoid post-operative pain.

\section{References}

1. Bettinger PC, Linscheid RL, Berger RA, Cooney WP 3rd, An $\mathrm{KN}$. An anatomic study of the stabilizing ligaments of the trapezium and trapeziometacarpal joint. J Hand Surg Am. 1999; 24:786-798.

2. North ER, Rutledge WM. The trapezium-thumb metacarpal joint: the relationship of joint shape and degenerative joint disease. Hand. 1983;15: 201-206.

3. Ateshian GA, Rosenwasser MP, Mow VC. Curvature characteristics and congruence of the thumb carpometacarpal joint: differences between female and male joints. J Biomech. 1992;25:591-607.

4. Mrzke MW, Tocheri MW, Marke RF, Femiani JD. Threedimensional quantitative comparative analysis of trapezialmetacarpal joint surface surface curvatures in human populations. J Hand Surg Am. 2012;37:72-76.

5. Pellegrini VD Jr, Olcott CW, Hollenberg G. Contact pat- terns in the trapeziometacarpal joint: the role of the palmar beak ligament. J Hand Surg Am. 1993;18:238-244.

6. Pellegrini Jr VD. Osteoarthritis of the trapeziometacarpal joint: the pathophysiology of articular cartilage degeneration. II Articular wear patterns in the osteoarthritic joint. J Hand Surg Am. 1991;16: 975-982.

7. Iameda T, An KN, Cooney WP 3rd, Linscheid R. Anatomy of trapeziometacarpal ligaments. J Hand Surg Am. 1993;18:226-231.

8. Edmunds JO. Current concepts of the anatomy of the thumb trapeziometacarpal joint. J Hand Surg Am. 2011;36:170-182.

9. Zhang AY, Van Nortwick S, Hagert E, Ladd AL. Thumb carpometacarpal ligaments inside and out: a comparative study of arthroscopic and gross anatomy from the Robert A. Chase Hand and Upper Limb Center at Stanford University. J Wrist Surg. 2013;2:55-62.

10. Vitale MA, Taylor F, Ross M, Moran S. Trapezium prosthetic arthroplasty (silicone, artelon, metal, and pyrocarbon). Hand Clin. 2013;29:37-55.

11. Swanson AB, deGoot Swanson G, Watermeier JJ. Trapezium implant arthroplasty. Long-term evaluation of 150 cases. J Hand Surg Am. 1981; 6:125-141.

12. Mac Dermid JC, Roth JH, Rampersaud YR, et al. Trapezial arthroplasty with silicone rubber implantation for advanced osteoarthritis of the trapeziometacarpal joint of the thumb. Can J Surg. 2003;46:103-110.

13. Minamikawa Y, Peimer CA, Ogawa $R$, et al. In vivo experimental analysis of silicone implants on bone and soft tissue. J Hand Surg Am. 1994;19:575-583.

14. Pellegrini VD, Burton RI. Surgical management of basal joint arthritis of the thumb. Part I. Long-term results of silicone implant arthroplasty. J Hand Surg Am. 1986;11:309-324.

15. Burton RI, Pellegrini VD. Surgical management of basal joint arthritis of the thumb. Part II. Ligament reconstruction with tendon interposition arthroplasty. J Hand Surg Am. 1986;11:324-332.

16. Lehmann O, Herren DB, Simmen BR. Comparison of tendon suspension-interposition and silicon spacers in the treatment of degenerative osteoarthritis of the base of the thumb. Ann Chir Main Memb Super. 1998;17:25-30.

17. Regnard PJ. Electra trapezio metacarpal prosthesis: results of the first 100 cases. J Hand Surg Br. 2006;31:621-628.

18. Chakrabarti AJ, Robinson AH, Gallagher P. De la Caffinière thumb carpometacarpal replacements. 93 cases at 6 to 16 years follow-up. J Hand Surg Br. 1997;22:695-698.

19. August AC, Coupland RM, Sandifer JP. Short term review of the De la Caffinière trapeziometacarpal arthroplasty. J Hand Surg Br. 1984;9:185-188.

20. Bellemère P, Gaisne E, Loubersac $T$, et al. Pyrocardan implant: free pyrocarbon interposition for resurfacing trapeziometacarpal joint. Chir Main. 2011;30: S28-S35.

21. Stabler D. Pyrodisk trapezium replacement. Abstract book Australian Hand Surgery Society Annual Meeting. Bunker Bay, Australia. 2011.

22. De Smet L, Sioen W, Spaepen D, et al. Total joint arthroplasty for osteoarthritis of the thumb basal joint. Acta Orthop Belg. 2004;70:19-24.

23. Cooney WP, Linscheid RL, Askew LJ. Total arthroplasty of the thumb trapeziometacarpal joint. Clin Orthop Relat Res. 1987; 220:35-45.

24. Igoe D, Middelton C, Hammert W. Evolution of basal joint arthroplasty and technology in hand surgery. Journal of Hand Therapy. 2014;27:115-121. 